Wien klin Mag 2021 · 24:213

https://doi.org/10.1007/s00740-021-00422-z

Angenommen: 8. November 2021

(C) The Author(s), under exclusive licence to Springer-Verlag GmbH Austria, ein Teil von Springer Nature 2021

Träume haben in unserer rationalen $\mathrm{Ge}$ sellschaft oft kein großes Ansehen. Sie werden als realitätsfern, zu emotional oder wenig zielführend betrachtet. Das ist ein ziemlich kurzsichtiges Pauschalurteil. Martin Luther Kings emphatisch vorgetragener Traum einer USA, in der soziale, ökonomische, politische und rechtliche Gleichstellung der Afroamerikaner verwirklicht wäre, liegt auch schon lange zurück und ist überdies bis heute nicht erfüllt. Das Potenzial des Träumens in all seinen Facetten als Erweiterung der Wirklichkeit liegt schon geraume Zeit ziemlich brach. Aber es gibt durchaus Einzelbereiche, wo Umbrüche zu bemerken sind - und es sind die Kreativen und die Jungen, diehier erfreulich frische oder zumindest aufgefrischte Wege gehen.

\section{Ein durchgängiges Prinzip}

Naturgemäß bildeten Träume auch beim Filmfestival Viennale im vergangenen Herbst so etwas wie ein durchgängiges Prinzip: Nachtträume, Tagträume, Träume als Wunschvorstellungen und als Flucht vor der Wirklichkeit ... In ihrem Film „Land of Dreams“ hat beispielsweise die iranisch-stämmige Künstlerin und Filmregisseurin Shirin Neshat gemeinsam mit ihrem Landsmann Shoja Azari eine reizvolle Doppelbödigkeit eingebaut. Nicht nur schicken sie die Protagonistin - mit autobiographischen Zügen - im Auftrag des US Zensusbüros auf eine Erkundungstour, um die Nachtträume der Bevölkerung zu erfragen und dokumentieren, sondern sie zeigen mit diesem Erzählstrang, mit dem sie in die privaten Lebensumstände der Menschen führen, auch die Vielschichtigkeit ihrer neuen Heimat und der

Verena Kienast

Springer Medizin, Wien, Österreich

\title{
Plädoyer für Träume
}

\section{Offen für die Vielfalt des Möglichen}

Ausprägungen des „American Dream“ auf. Die Überlegung, Träume offiziell erfassen zu lassen, lässt freilich ebenso zahlreiche Gedankenspiele $\mathrm{zu}$ - von poetischer Lebensbegleitung bis zum Überwachungsstaat.

\section{Chronologische Sprünge}

Terence Davis, der besonders unter Kritikern hochgeschätzte britische Filmregisseur und Ehrengast der Viennale 2021, verarbeitet in seinem nicht sehr zahlreichen, dafür aber umso prägnanteren Werk seine eigene schwierige Lebensgeschichte ebenso, wie schwierige Lebensgeschichten zum Teil historischer Persönlichkeiten. In seinem neuesten Film ist das der britische Dichter und Erzähler Siegfried Sassoon, der seine traumatischen Erfahrungen des Ersten Weltkriegs bis an sein Lebensende nicht bewältigte. Oft sind es chronologische Sprünge, die in ihrer unaufgeregten Betrachtungsweise von durchaus auch gewaltsamen Ereignissen, von Träumen und Albträumen und der Hilflosigkeit gegenüber Ausgrenzung und Gewalt im Kleinen wie im Großen, die komplexen, freud- und leidvollen Entwicklungsgeschichten einfühlsam nachvollziehbar machen.

So wie Literatur und bildende Kunst, zeigt und eröffnet der Film mit der Thematisierung des Traums als gleichwertiger Realität die Vielfalt der Möglichkeiten: Im Guten wie im Bedrohlichen. Das Potenzial, den Geist zu beflügeln und $\mathrm{Ge}$ danken zuzulassen, die im starren System des Alltags oft keinen Platz haben, sollte man freilich mit Bedacht nützen oder man kann sich auch der Faszination der Traumwelt genussvoll hingeben.

\section{Im Filmtraum sicher vereint}

Die erfreuliche Realität der Großveranstaltung Viennale unter der souveränumsichtigen Leitung von Eva Sangiorgi: So wie auch im Jahr davor ist es heuer wieder gelungen, das Festival unter geordneten Corona-Bedingungen erfolgreich über die Bühne zu bringen, mit gründlichen Kontrollen und disziplinierten, im Filmtraum begeistert vereinten Teilnehmern. Ein gutes Vorbild in jeder Hinsicht

meint Ihre

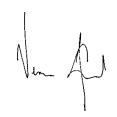

\section{Korrespondenzadresse}

\section{Verena Kienast}

Springer Medizin

Wien, Österreich

verena.kienast@springer.at

Hinweis des Verlags. Der Verlag bleibt in Hinblick auf geografische Zuordnungen und Gebietsbezeichnungen in veröffentlichten Karten und Institutsadressen neutral. 